\title{
Automatic Pre-Cooling System for Large Infrared Instruments
}

\author{
Koji Omata*a ${ }^{*}$ Tetsuo Nishimura ${ }^{a}$, Stephen Colley ${ }^{\mathrm{a}}$, David Cook ${ }^{\mathrm{a}}$, William Gorman ${ }^{\mathrm{a}}$, \\ Barney Magrath ${ }^{\mathrm{a}}$, Lucio Ramos ${ }^{\mathrm{a}}$, Scot Kleinman ${ }^{\mathrm{b}}$, Chihiro Tokokuc ${ }^{\mathrm{c}}$, Masahiro Konishi ${ }^{\mathrm{a}}$, \\ Tomohiro Yoshikawa ${ }^{a}$, Ichi Tanaka ${ }^{a}$, Ryuji Suzuki ${ }^{a}$ \\ ${ }^{a}$ National Astronomical Observatory of Japan, 650 North A'ohoku Place, Hilo, HI, USA 96720; \\ ${ }^{\mathrm{b}}$ Gemini Observatory, 670 North A'ohoku Place, Hilo, HI, USA 96720; \\ ${ }^{\mathrm{c}}$ Astronomical Institute, Tohoku University, Aramaki, Aoba-ku, Sendai, Japan 980-8578;
}

\begin{abstract}
An infrared instrument used for observation has to keep the detector and optical components in a very cold environment during operation. However, because of maintenance, upgrades, and other routine work, there are situations that require the instrument to be warmed-up and then cooled-down again. At Subaru Observatory, our MOIRCS infrared instrument has required warm-up and cool-down several times a year for routine maintenance and filter replacement. The MOIRCS instrument has a large heat capacity and cool-down using only the closed cycle cooler is impractical due to the huge amount of time it would require. To address this problem Subaru engineers have created a mechanism to allow PRECOOLING of the instrument via liquid nitrogen - allowing for a much faster pre-cool process. Even with liquid nitrogen, the pre-cool process requires 10 tanks and almost a week of continual monitoring in order to reach the desired target temperature. It is very difficult to work for such a long period of time at the oxygen starved summit of Mauna Kea (4205 meters),and issues of man-power and scheduling conflicts only add to the problems. To address these concerns Subaru developed an automated pre-cooling system which works continuously and remotely at the summit. The strategy was to have basic functionality for pre-cooling and user friendly interface. i.e. (1) Continuous cooling until the target temperature is reached by automated liquid nitrogen tank exchanges and precision temperature control by automated changes to the liquid nitrogen flow. (2) Remote monitoring and control of all parameter setting by Web browser as user interface (UI). The goal of the Subaru pre-cooling system was to make it both inexpensive and quick to implement by using existing technologies. The original goal (to cut down on labor and precision temperature control) has been attained through several pre-cooling and software/hardware modification cycles. We will report on the progress and status of our pre-cooling experiences in this presentation.
\end{abstract}

Keywords: control, infrared, instruments, cryogenic, operation, web application, subaru, moircs

\section{INTRODUCTION}

The development of the Automatic Pre-cooling System (APS) was motivated by the task of cooling MOIRCS (Multi-Object InfraRed Camera \& Spectrograph) ${ }^{[1]}$ now in operation on the Subaru Telescope. The goal of APS, however, is to make the system available to all Subaru instruments in order to increase the safety of expensive and sophisticated instruments and at the same time to reduce the load of the labor-intensive task to the operation team.

The entire optics of MOIRCS is cooled in a large cryostat (Fig. 1) to extend the band coverage from J- to $\mathrm{K}$-band in near infrared. The cooled system includes two sets of ten infrared lenses each, including $210 \mathrm{~mm}$ diameter $\mathrm{CaF} 2$ lenses, filters and grisms and two large 2048 × 2048 pixel Hawaii-2 arrays. In addition, a unique cooled slit-mask exchanger system is located at the focal plane of the telescope just inside of a large $\mathrm{CaF} 2$ window at the top of the cryostat.

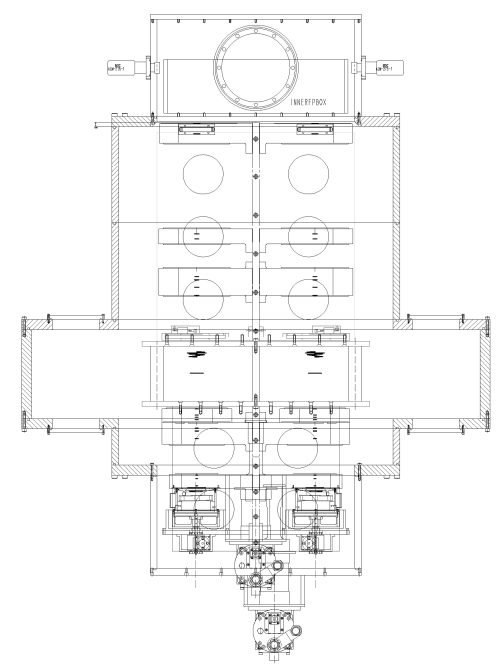

Fig. 1. MOIRCS drawing. The height of cryostat is about $1600 \mathrm{~mm}$. The weight of contents is $300 \mathrm{~kg}$. 
In the past, the pre-cooling of many instruments has been made in some cases without much control of the temperature rate. However, most careful control is required for large instrument such as MOIRCS because there were incidents that too fast temperature decrease caused damage to the optics or infrared detectors. The reason we need very careful temperature control in the cooling cycle of large infrared instruments such as MOIRCS are as follows:

\section{Thermal stress}

The infrared lens materials have low thermal conductivity. Rapid temperature change of large optics makes stress exceeding the yield stress and possibly induces cracks. Besides, since the lenses are mounted in contact with the metal parts directly, and cooling rate too fast or uncontrolled might make uncontrolled friction between a lens and lens mount due to the difference of thermal contraction, eventually the lens may be damaged.

\section{Condensation on the detector and optics}

If condensation occurs on the detector, a short circuit could break out on the surface of the detector and the detector could be destroyed. If condensation occurs on the lenses, the optical performance will be impaired significantly by frost over the lenses. These parts should be kept at highest temperature in the dewar during pre-cooling. The condensation will occur on other parts where temperature is lower than the detectors and optics. Since the detectors are equipped with controlled heater and the thermometer, it can recover from the condensation by using heater. In case of the optics, if the dew condensation occurs at cold environment, it can not be removed until the dewar will be warmed up to higher temperature. The major gases that may condense on cooled surfaces are water vapor, carbon dioxide, oxygen and organic molecules. Thermal control of the detectors and optics should be done most carefully to maintain the temperature below their vapor pressure curves as illustrated in Fig. 2.

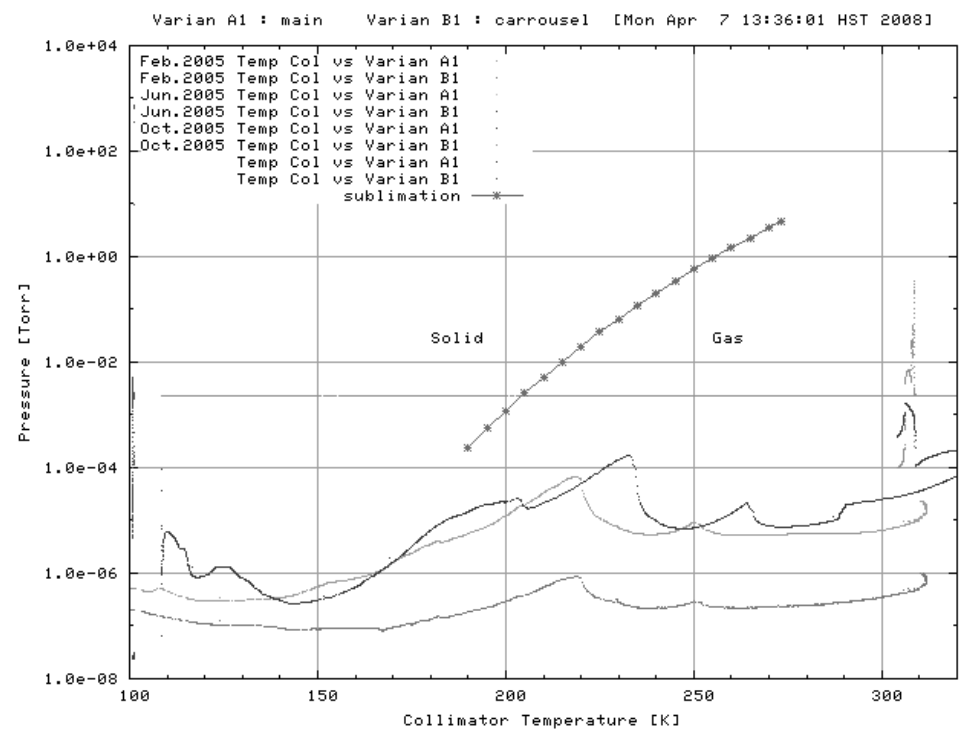

Fig. 2. Temperature versus pressure curve for water vapor. A line with dots represents a water vapor curve. Collimator (optics) temperature is shown to stay below the vapor curve. This prevents the formation of a water condensation.

The Subaru instruments usually have several thermometers, a closed cycle cooler or two, liquid nitrogen cooling pipeline, electric heaters inside the dewar. The cooling process is done by taking advantage of these sensors and actuators and advanced control software.

During the development phase of MOIRCS, pre-coolings were done manually. Many staff members were involved and 24 hour continuous watch was at sea level. (i.e., laboratories in the HILO base of the Subaru Telescope.) As the work shifted to Mauna Kea summit, the manpower shortage became considerable. The person on duty had not only to stay in the thin air and cold environment in the dome at the summit but also to sustain concentration for watching status of devices and controlling temperature. Because of the manual valve for liquid nitrogen flow control, tank replacement of empty liquid nitrogen tank and vacuum pumping of the cooling line after the pre-cooling had to be done on site in spite of the most of status were obtained remotely. In addition, work is not allowed during nighttime in the dome where 
instruments are located. Pre-cooling work at the summit is intense, but not effective because of nighttime interruption. Such an intermittent cooling process is not good for instruments and wastes liquid nitrogen. So the automated precooling, which can do it 24 hour continuously, was necessary.

There are relevant technologies in aerospace controlling a cryogenic fluid flow, (i.e. rocket engine control with liquid fuel and oxidant), metal industry, food industry, chemical industry, biochemical area, medical, nuclear physics, etc. We investigated commercial product of liquid nitrogen cooling system with precise temperature rate control but did not find any system meeting exactly our demand. So we decided to build a new Automatic Pre-cooling System (APS) by combining commercial cryogenic components such as control valve, electric valve and tank switcher.

For the development of APS, three control systems are required; (1) liquid nitrogen flow control, (2) liquid nitrogen tank switching and (3) vacuum pumping after the pre-cooling. The first goal was set for complete remote control of the precooling with liquid nitrogen tank transportation and connection to the system at most once a day. The second goal was set for cooling down with a given temperature rate and smooth tank switching. Also establishing the procedure and easy operation for those with minimum training was also the goal. The development took a few years because the testing opportunity during maintenance of such large instruments is only twice or three times per year.

\section{MANUAL PRE-COOLING}

This part describes the components, equipment and method of pre-cooling before it was automated. This helps to understand of basic operation of the pre-cooling as applied to MOIRCS.

\subsection{Cooling line}

For pre-cooling the instrument by liquid nitrogen, a cooling line is installed inside the dewar as shown in Fig. 3. A section of the cooling line in contact with the optical bench that contains the detector, optics, filters, will be made of material having higher heat conductivity such as copper. The cooling line passes a heat exchanger panel to maximize heat transfer. Inlet and outlet ports at the dewar wall are made of material having lower heat conductivity such as stainless steel in order to insulate the liquid nitrogen from the ambient temperature. After the pre-cooling, the cooling line will be pumped out to remove the residual coolant in the pre-cooling line and sealed.

\subsection{Liquid Nitrogen}

Liquid nitrogen is delivered in conventional storage vessels. The stainless steel container with double-walled vacuum insulation vessel has capacity of 150 170 liter. The inner vessel is sealed and pressurized by pressure building. The vaporizer makes nitrogen gas by contacting liquid nitrogen to ambient temperature. The pressure is regulated within several tens of psi and excess pressure is released by relief valves. This pressure presses down the liquid, which comes out through a liquid withdrawal pipe. A natural evaporation rate

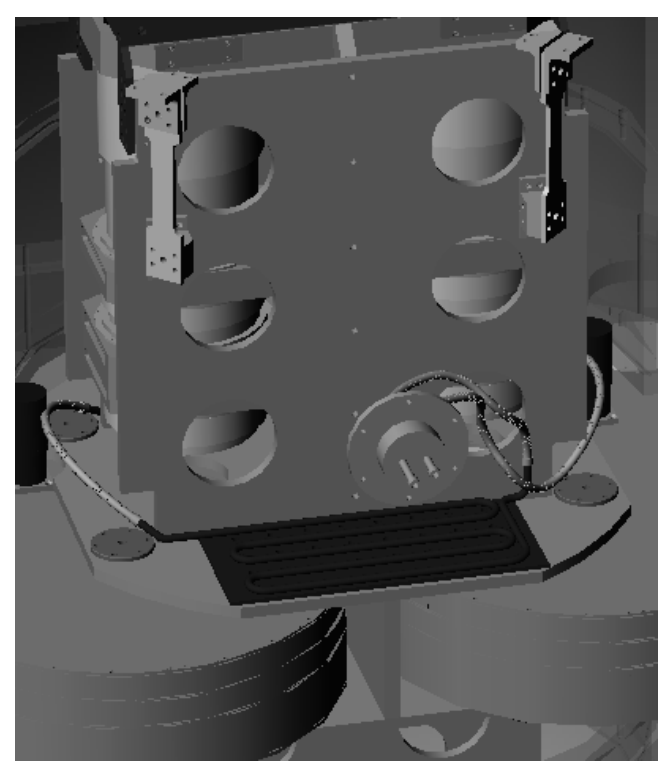

Fig. 3. An example of pre-cooling line. This is 3D image of inside of MOIRCS dewar. The cooling line is installed near the center of the dewar contents. Turret units shown as expanding cylinders have largest amount of heat capacities. The cooling path is installed where cooling is most effective. of liquid nitrogen in the storage container is about $2 \sim 3 \%$ per day. As the liquid level lowers, pressure building supplies more gas by vaporizing the liquid to maintain the inner pressure. If the liquid runs out, that pressure will rapidly drop down. Amount of the liquid nitrogen to cool from room temperature to cryogenic temperature is ideally supposed to be less than $30 \%$ of mass to be cooled. ${ }^{[2]}$ (About 100 litter for MOIRCS). However, for pre-cooling liquid nitrogen is consumed more than ideal amount because of heat waste along the cooling line in the system. It is hard to estimate the amount of liquid nitrogen actually required. 


\subsection{Closed Cycle Helium Cooler}

MOIRCS has two cryogenic closed cycle cooler coldheads (Sumitomo RDK-408S). One is directly connected to the optical bench which keeps the optical bench at cryogenic temperature. However, its cooling power of $80 \mathrm{~W}$ alone would take more than a few weeks to reach the target temperature. It does help the pre-cooling much with liquid nitrogen, however. The second coldhead is for cooling slit masks which are contained in a carrousel container that can be isolated by a gate valve. It has frequent cycle of warm-up and cool-down to exchange the masks for specific observations and it is not connected thermally to the optical bench. This is used as a powerful cryo-pump during cooling-down phase. There are molecular sieve baskets installed in near the cooling heads to adsorb residual or out-gas.

\subsection{Measurement and Control Devices}

\section{Thermometer}

An infrared instrument will have its own cryogenic thermometers in the dewar to control the inside temperatures. MOIRCS has eight temperature sensors. These are connected through two sets of temperature controllers to the instrument computer. The temperature controller controls heaters to maintain the temperature of the detector arrays during the pre-cooling and the operation.

\section{Flow meter}

A flow meter initially used was for room temperature operation. It was put at the end of exhaust rubber tube to measure a gas flow rate. The measurement was not reliable because (1) liquid nitrogen occasionally reached to the flow meter and made the flow reading extremely low, (2) the flow meter has a rotating screw fin that oscillated at a certain rotation speeds, making the flow reading drop. So the flow meter was merely used for confirmation of flow. A cryogenic flow meter using pressure difference did not work well because the tube friction resistance was higher and broke easily. So the flow meter for liquid nitrogen has been never used in this system.

\subsection{Operation}

Pre-cooling operation of MOIRCS is described as follows.

\section{Preparation of liquid nitrogen tank}

Usually seven tanks liquid nitrogen are sufficient for manual cooling. The liquid nitrogen tank of is connected to the pre-cooling line by insulated hoses, but connecters or joints are exposed. The exit port of the pre-cooling line is connected to a long rubber hose leading to outside of the workspace to prevent depleting oxygen with exhaust nitrogen gas. Other sensors like a flow sensor can be installed in the cooling line if it is required.

2. Flow control by manual valve

The flow rate will be unstable initially until the liquid nitrogen cools down the cooling line sufficiently. The nitrogen gas is let out for a while if required. The coolant flow at the beginning is very sensitive to the valve control. Precise control is required at the beginning of

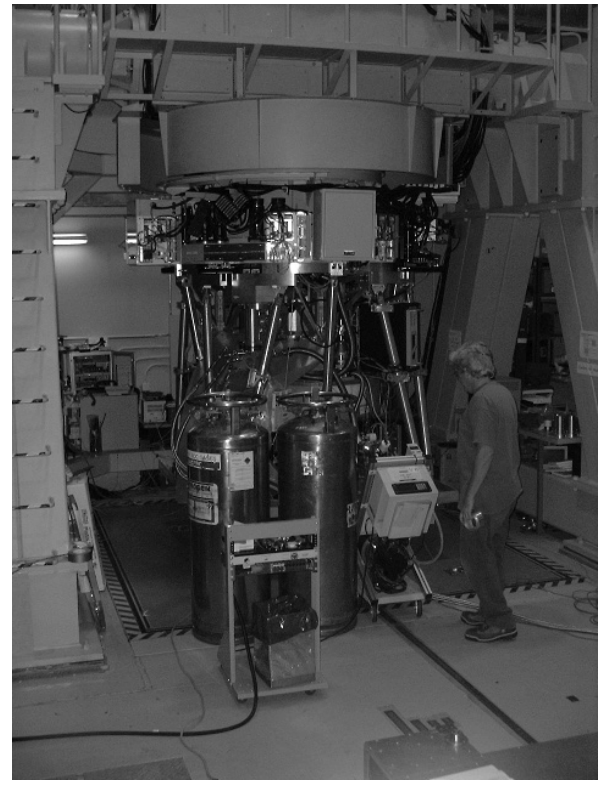

Fig. 4. Manual pre-cooling at Simulatorlab at the Subaru base. The operator must be there for 24 hours for watching the system. the pre-cooling. The control of the valve knob to set a constant flow rate might depend on the ambient temperature and valve backlash.

\section{Temperature control}

The relationship of the liquid nitrogen flow rate and temperature drop rate is non-liner, and has delay time of several minutes. The valve has to be controlled carefully even if the current temperature misses the target rate because of the delay time. The accuracy of the temperature control depends on the skills of the operators. The specified cooling rate is less than $5 \mathrm{~K}$ per hour. The target of temperature in MOIRCS is in Table. 1. 
Table. 1. Stabilized temperature and target temperature of MOIRCS. The target temperatures of pre-cooling are set below the stabilized temperatures. The first column gives names various point inside MOIRCS.

\begin{tabular}{|l|c|c|c|}
\hline \multirow{2}{*}{} & \multicolumn{2}{|c|}{ Stabilized temperature } & \multirow{2}{*}{ Goal of Pre-cooling } \\
\cline { 2 - 3 } & Summer & Winter & \\
\hline RoboHand & $122 \mathrm{~K}$ & $116 \mathrm{~K}$ & $110 \mathrm{~K}$ \\
\hline CollBench & $105 \mathrm{~K}$ & $100 \mathrm{~K}$ & $90 \mathrm{~K}$ \\
\hline BulkHead & $106 \mathrm{~K}$ & $102 \mathrm{~K}$ & $90 \mathrm{~K}$ \\
\hline HeatPath & $88 \mathrm{~K}$ & $88 \mathrm{~K}$ & $80 \mathrm{~K}$ \\
\hline
\end{tabular}

\section{Tank replacement}

When the tank becomes empty, rapid decrease of the temperature rate is observed. After the tank replacement, the gas from a new tank should be vented out for a while because of high temperature at the beginning. Even so, a certain amount of the gas comes into the cooling line and makes temperature rise in the dewar.

\section{Termination}

When all the tanks run out or halt of the pre-cooling is necessary, the cooling line has to be pumped out by vacuum pump. This is only to remove any residual nitrogen in the pre-cooling line, and a scroll pump is sufficient.

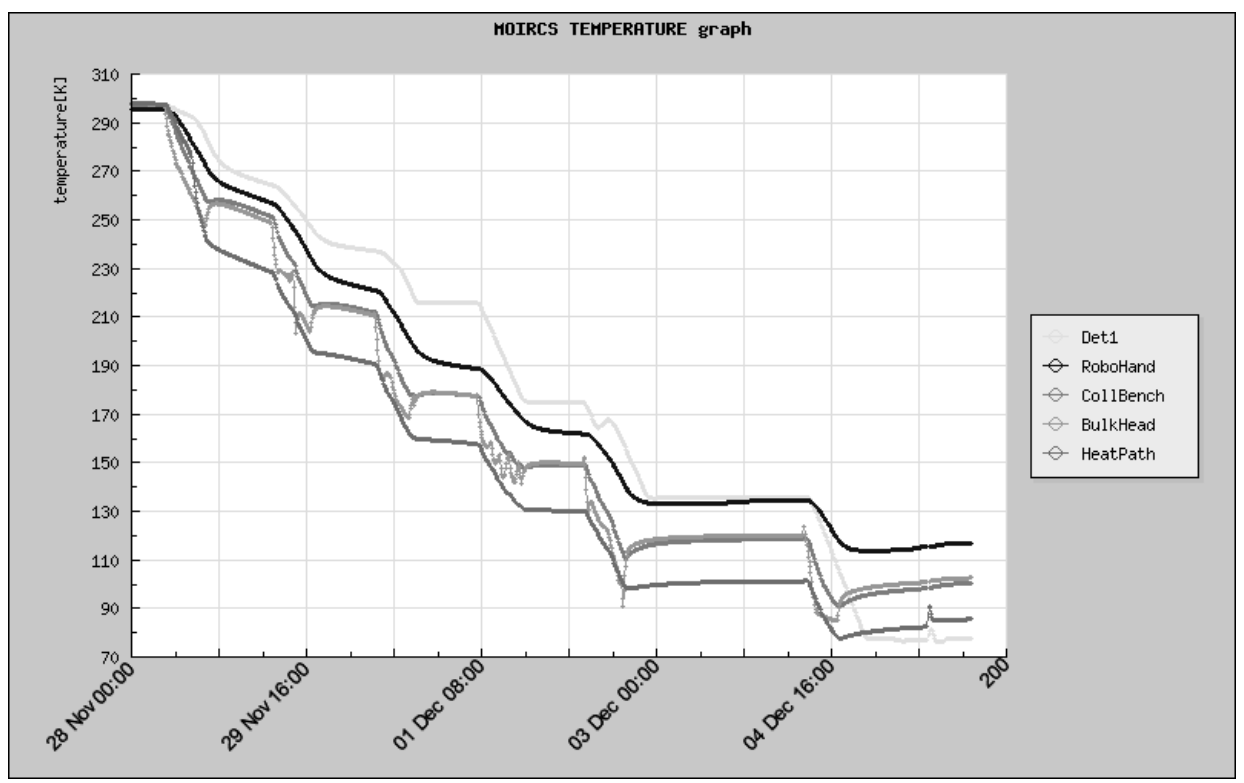

Fig. 5. An example of temperature curve of pre-cooling at the summit. In the manual pre-cooling, working hours are restricted to daytime, so the curve shows a step pattern. The helium closed cycle keeps temperature constant during night. BulkHead is nearest to the pre-cooling line and the change in temperature is most sensitive to the liquid flow. CollBench temperature represents the optics whose rate must be controlled precisely. The detectors are maintained at highest temperature in the dewar during pre-cooling. 


\section{CURRENT SYSTEM}

The current APS is divided into a control system and a liquid nitrogen supply system. The control system contains almost all the active control devices, monitoring sensors, control computer and connections to the instrument. The liquid nitrogen supply system has the coolant supply tanks and a liquid nitrogen tank which supplies nitrogen gas to the supply tanks to maintain a constant pressure in all tanks.

\subsection{Control System}

A block diagram of the current control system is shown in Fig. 6. Identification of the major elements in the coolant path is in the figure caption. The electronics for the control system are in a rack which also holds the control valve and many other coolant path components Electric valves use line voltage and are controlled via networked power switches over a local area network (LAN). Pressure and temperature sensor readouts communicate with the control computer over standard serial connections using a terminal server on the LAN. The control computer is located at the Subaru operations center in Hilo, Hawaii and uses the Subaru network to the summit of Mauna Kea. The system is controlled by operators logged into the control computer using standard web browsers over a LAN or from anywhere using the Internet. The system is monitored at all times by an operator. Evening and late night shifts are typically performed by members of the APS team operating from home.

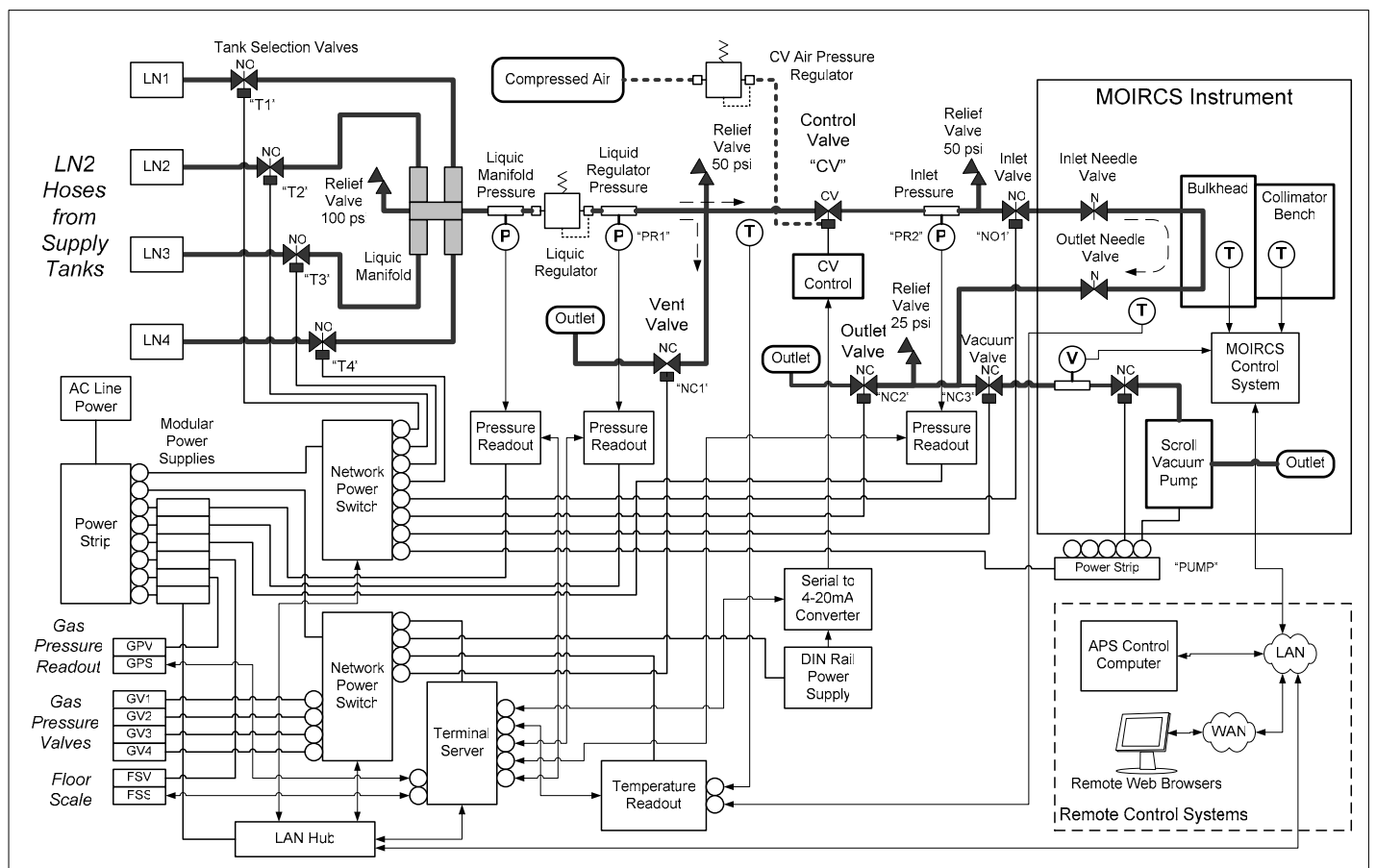

Fig. 6. Current APS Control System Block Diagram. Thick solid lines carry coolant. A liquid nitrogen tank is selected by electric valves T1 to T4. Incoming coolant pressure is regulated with a fixed regulator. The proportional Control Valve sets the flow of liquid nitrogen under control of the Control Computer. Instrument bulkhead and collimator temperatures are read by the Control Computer over a local network from the instrument Control System. The Control Computer is monitored and controlled from web browsers over local or wide area networks. The Vent Valve provides a momentary path for warm gas immediately after a tank switch. The Scroll Vacuum Pump removes any gas trapped between the instrument Inlet Valve and the Outlet Valve after pre-cooling. The Inlet and Outlet Needle Valves on the instrument are fully open during pre-cooling with the APS.

\subsection{Liquid Nitrogen Supply System}

A block diagram of the current liquid nitrogen supply system is shown in Fig. 7. Identification of the major elements for the coolant path and gas pressure control is in the figure caption. Experience with previous versions of the system showed the necessity of maintaining a constant pressure in the liquid nitrogen supply. Flow through the control valve is a 
strong function of pressure across the valve. This means variation in the supply pressure is effectively a change in the feedback control system gain. In addition, we use nitrogen tanks from a local supplier of cryogenic products. The tanks vary considerably in their pressure building characteristics. For these reasons, we purchased our own tank for dedicated use in maintaining constant and equal pressure in all supply tanks. This is done by connecting all the gas vent ports on the tanks through a manifold. Electric valves to each supply tank gas port allow empty tanks to be isolated from the pressure system. A floor scale is used to continuously monitor the remaining nitrogen in the pressure tank.

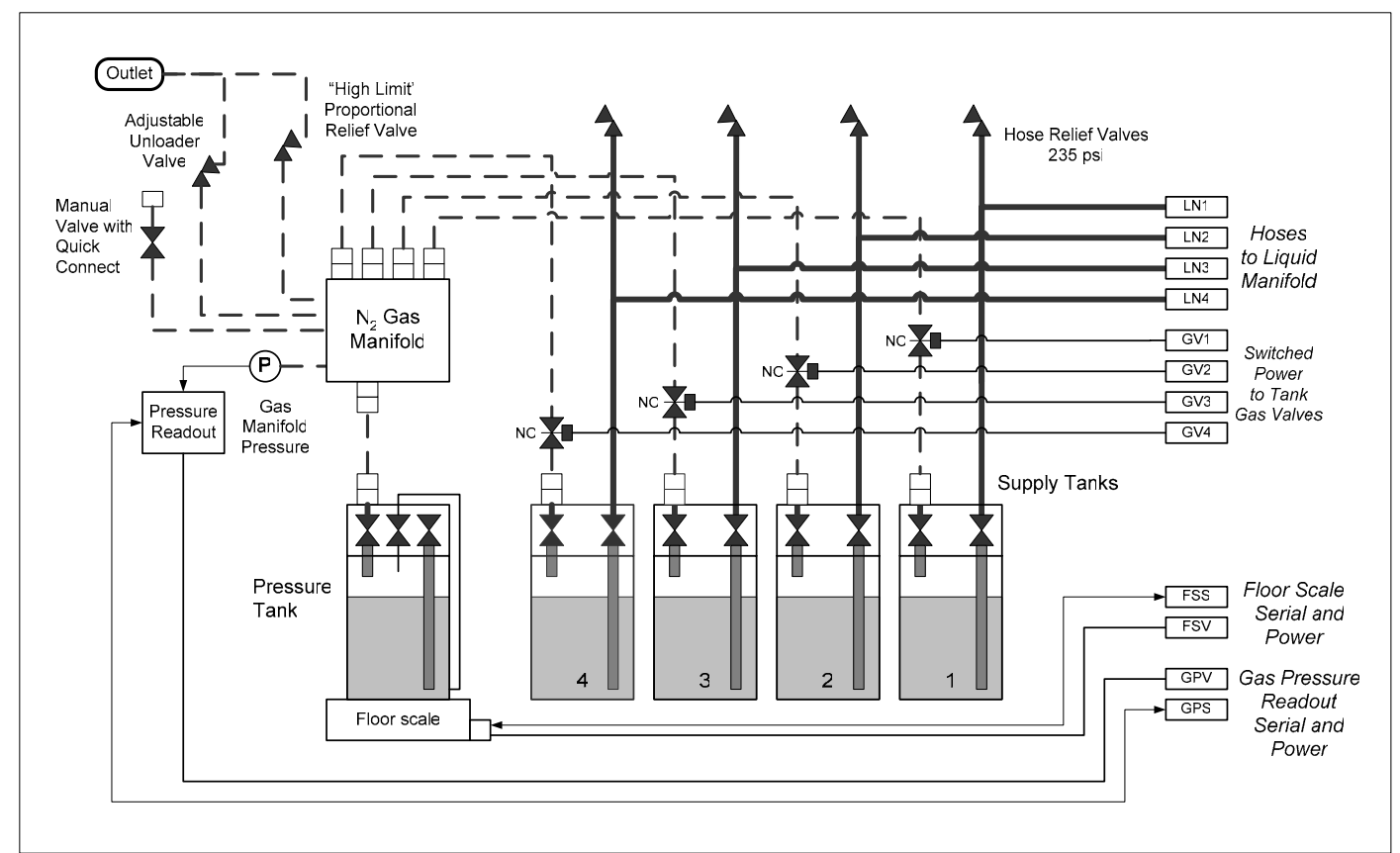

Fig. 7. Liquid Nitrogen Supply System Block Diagram Thick solid lines carry coolant. Dotted lines contain nitrogen gas for maintaining constant and equal pressure in all tanks. The liquid ports of four liquid nitrogen storage tanks connect to four supply hoses to the control system. The gas port of each supply tank connects to a gas manifold through an electric valve. A separate liquid nitrogen tank connects to the gas manifold and is dedicated to controlling the pressure in the supply tanks using internal pressure building. A Proportional Relief Valve sets a high limit to the pressure in the system if the pressure tank builds higher than normal. A Floor Scale monitors the amount of liquid nitrogen in the Pressure Tank which must not become empty. Gas Manifold pressure is monitored to check for the correct range of pressure. The Adjustable Unloader Valve is for safety and does not normally activate. The Manual Valve allows for release of pressure and connection of an additional source of gas pressure during testing.

\subsection{Tank Switching and Venting}

A critical issue is how the control computer initiates a tank switch when a supply tank is empty. One approach is to have a floor scale on each supply tank and simply use weight to determine remaining liquid nitrogen. However, the current system monitors supply pressure and applies a predictive algorithm to detect loss of pressure when a tank is empty. The algorithm must differentiate between an empty tank and pressure variations during normal operation. If the pressure is allowed to drop too far, warm gas is allowed into the instrument and pressure in the liquid nitrogen supply system takes longer to recover. The current system can reliably detect pressure drops of $10 \%$ in nominal supply pressure to start the tank switch procedure.

After a tank switch there is a period of time when warm gas flows from the new tank until the new supply hose frosts over and stabilizes. Immediately after opening the valve to the new tank the system performs a venting operation. This operation prevents warm gas from entering the instrument and reduces the time needed for the new coolant path to stabilize by allowing a large flow of gas and liquid to an outlet hose. During venting, the control computer monitors the temperature of the pipe to the outlet. The temperature initially increases when gas is flowing and decreases quickly with 
a flow of liquid nitrogen. A pipe temperature of $105 \mathrm{~K}$ was determined empirically to reliably indicate a return to normal liquid nitrogen flow. At this point the system returns to automatic cooling.

\subsection{Operating modes}

There are four main operating modes: "User", "Cooling", "Pumping” and "Tank Replacement".

1. Cooling mode

This mode automatically cools the instrument at a set rate of cooling. The control system watches the coolant supply pressure to detect an empty tank and will automatically switch to a new tank. The system opens the Vent Valve to release warm gas from the new tank and prevent warming of the instrument. Venting continues until the coolant supply temperature drops to indicate flow of liquid nitrogen at which point normally cooling resumes. The system maintains the status of all tanks and will go to pumping mode if all tanks are emptied.

\section{Pumping mode}

If all tanks empty in Cooling mode, the system goes to Pumping mode. The inlet and outlet valves are closed and the pump valve is opened. Finally, the vacuum pump is started. Pumping mode is also entered after return from a power failure or other problem conditions are detected. It is the final "safe" mode in any emergency.

\section{Tank Replacement mode}

An operator puts the system into Tank Replacement mode when empty nitrogen supply tanks are replaced. All the valves to the tanks are closed and it is safe to disconnect tanks. After replacing tanks, the operator completes a dialogue with the system to specify the new tank configuration. The system then returns to Cooling mode.

\section{User Mode}

User mode allows complete manual control of the system. This is used for testing and initial setup of the system before automatic cooling. In addition, User mode is used to recover from unforeseen events.

\subsection{Power Failure}

The system is carefully designed for safety in the event of a power failure. For example, during a power failure, the Outlet Valve and Vacuum Valve will close to prevent ambient air flow into the instrument. The Control Valve will close to isolate the instrument from the coolant source. Then, relief valves allow trapped nitrogen to evaporate and escape to prevent over pressures. After power returns, the system keeps the Control Valve closed and goes to "Pumping mode" and waits for an operator.

\subsection{Automatic Temperature Control}

The automatic cooling mode of the APS uses a control function shown in Fig. 8. A classic feedback loop controller maintains the instrument at a set temperature. The set point for the temperature control is continuously changed by a ramp generator which creates the cooling function as a series of regularly decreasing temperature steps. A typical ramp uses a $-1 \mathrm{~K}$ step every 12 minutes to produce a rate of $-5 \mathrm{~K}$ per hour. The ramp generator temperature is initialized to the current instrument temperature when switching from User to Cooling mode and after a tank switch or tank replacement. The feedback control has proportional, "P", and integral, "I", terms. Many parameters can be set to tune the performance of the temperature control (particularly the "I" term). Note the temperature controller can only actively cool the instrument using the liquid nitrogen control valve. The controller cannot actively warm the instrument. The only source of heat is natural, ambient heating. In practice, difficulties in controlling the "I" term under various conditions, has led to using only proportional control. This produces an offset between the set point and instrument temperature. However, absolute control of temperature is not critical as long as the rate of change is maintained. 


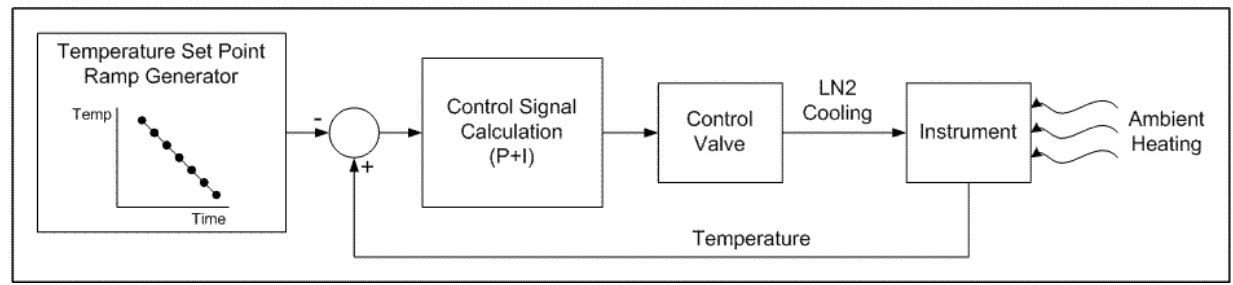

Fig. 8. Temperature Control Function. The rate of change in temperature is controlled by a ramp generator which continuously changes the temperature set point to a classic feedback control system. The APS can only cool the instrument. The only source of heat is the ambient environment. Temperature feedback is from the instrument control system and not a dedicated APS sensor.

\subsection{Future Improvement}

The current APS is highly dependent on the reliability of various networks. The control computer communicates with the cooling system over a dedicated network from the Subaru offices at the base of Mauna Kea and operators often use the Internet to monitor the system. Possible interruption in the base-to-summit network is particularly worrisome and could lead to an extended period with no manual or automatic control. Development of an embedded computer for the summit cooling system is underway to locally monitor the system and the network connection to the main control computer. If this "watch dog" function detects a network failure or other extreme condition, it will place the system in Pumping mode until an operator can intervene. Another option is to move all functions of the control computer to this embedded computer at the summit.

\section{RESULTS}

Fig. 9 contains graphs of the bulkhead and collimator bench temperatures during a pre-cooling run of the MOIRCS instrument in October of 2007. The collimator bench contains the optics which is sensitive to the rate of change in temperature. However, temperature control is applied to the bulkhead due to a much lower time constant from the cooling site to the bulkhead than from the cooling site to the collimator bench. This is possible because the collimator bench will follow and cannot change faster than the bulkhead. The lower time constant allows more accurate cooling and better stability in the control system. The setting for temperature rate of change in Cooling mode was $-5 \mathrm{~K} / \mathrm{Hr}$.

There is one importance difference between the current system and the system during the October 2007 run. The system in 2007 did not have the dedicated pressure building tank of the current system. Instead, Tank 4 was used for pressure building and provided coolant after the other three were exhausted. This situation required the pressure building tank to be replaced with a different tank each time there was a tank replacement. Problems with this configuration led to the addition of a fifth, dedicated pressure building tank.

The graph is divided with markers into eight intervals for the following detailed discussion. The caption contains definitions of the terms and abbreviations in the figure.

Interval 1-2: The system is started in manual or User mode. During this time, the control valve is set manually to allow the coolant path to "frost over" and stabilize. Brief periods of automatic, Cooling mode are run to set various control system parameters, primarily the gain setting for the proportional control term.

Interval 2-3: The system is running in automatic, Cooling mode. Two automatic tank switches occur before a tank replacement is performed at approximately noon of the second day. The system then continues to run in Cooling mode with four tank switches. All tanks are empty after the fourth tank switch and the system goes to Pumping mode. The shortened times before the final two tank switches are due to partially empty tanks. This caused coolant to run out before a tank replacement could be performed.

Interval 3-4: The system remains in Pumping mode until an operator arrives at the summit to perform a tank replacement. The total delay caused by running out of nitrogen is approximately 11 hours. The delay is defined as the time it takes to return to the same temperature when the system entered Pumping mode. The system was operated briefly in User mode at the end of Pumping mode to stabilize the system after it was off for a long period. 
Interval 4-5: The system operates in Cooling mode but problems with fluctuating tank pressure and control system instability cause confusion and the system is switched to User mode.

Interval 5-6: The control system starts to show instability and is switched to User mode for the remaining tanks. Then the system is put into Pumping mode until a tank replacement can be performed. The instability is due to a change in the thermal conductivity of copper in the cooling path at approximately $110 \mathrm{~K}$. The system does not have "zonal" control settings seen in some commercial cryogenic temperature controllers to change control parameters at different temperature regimes.

Interval 6-7: The system remains in Pumping mode until an operator arrives at the summit to perform a tank replacement with the remaining three, full tanks.

Interval 7-8: After the tank replacement, the system is operated in User mode with one manual tank switch just before midnight. During this interval, the bulkhead reaches the lowest practical temperature of about $90 \mathrm{~K}$. Cooling past this temperature requires large flows of liquid nitrogen with much of the liquid simply flowing through to the outlet and wasted.

Interval 8 - End: A successful experiment is run to operate in Cooling mode with a constant temperature setting of 90K. At this low temperature, the proportional gain is reduced to maintain stability. Pre-cooling ends when the collimator bench reaches $90 \mathrm{~K}$ and all the coolant is used.

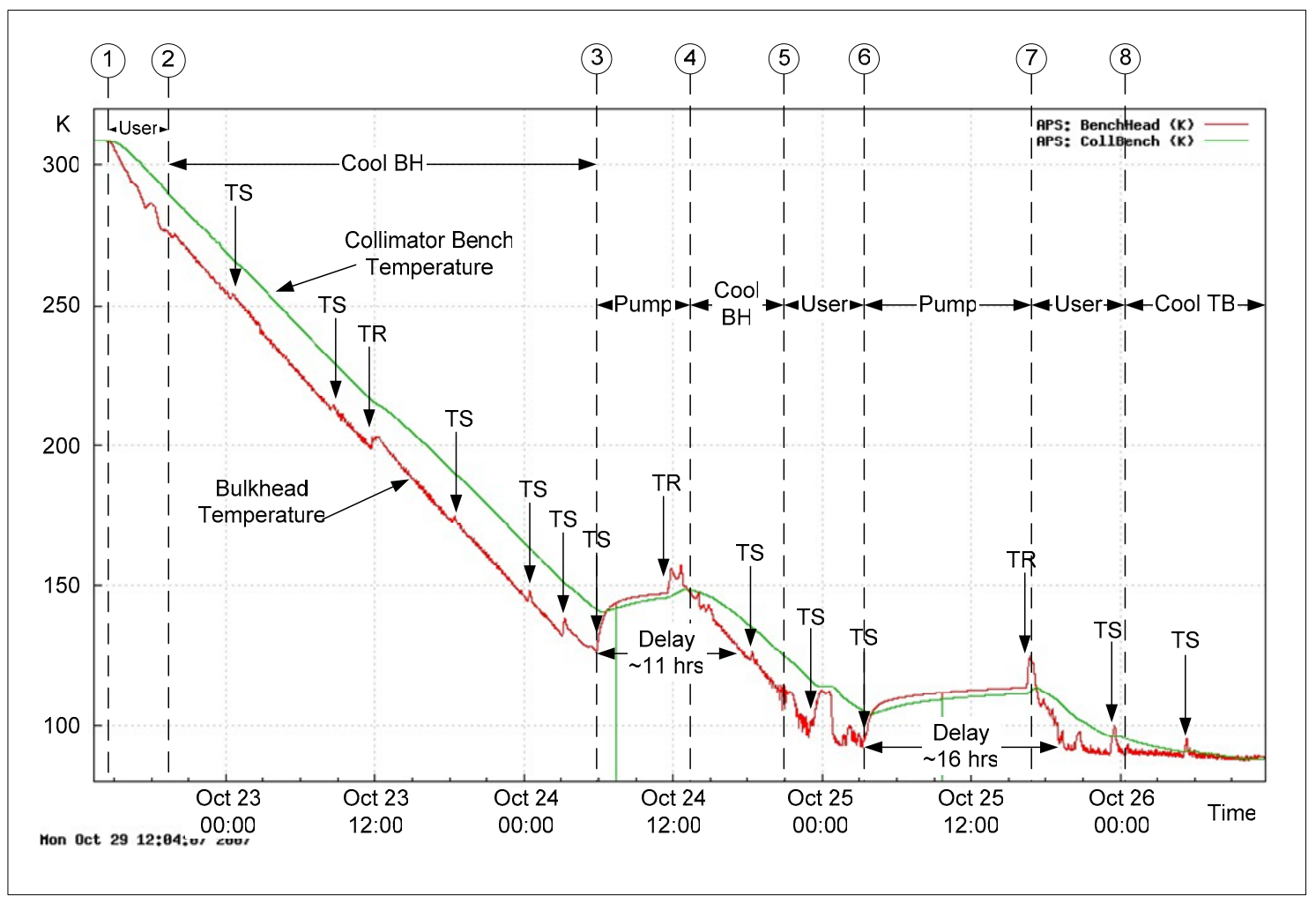

Fig. 9. Temperature curves from MOIRCS pre-cooling of October 2007. The figure shows bulkhead and collimator bench temperatures. The bulkhead was actively temperature controlled with the collimator bench lagging. "TS" is a Tank Switch event. "TR" is a Tank Replacement event. "User" is operation in User Mode. "Cool BH" is automatic Cooling mode with a temperature ramp applied to the bulkhead. "Cool TB" is Cooling mode with a constant temperature on the bulkhead. "Pump" is Pumping Mode. Delays are caused by periods of no cooling while waiting for tank replacements at the summit. 
Automatic tank switches followed by a short venting period are fast and efficient and cause little increase in temperature and subsequent delay in the pre-cooling time. Tank replacements cause larger increases in temperature but delays are small in relation to the overall pre-cooling time. Management of the liquid nitrogen supply is critical. Improvements include using only full tanks and better scheduling of tank replacement. It is important to avoid Pumping mode and the significant delays which can result from interruptions in cooling. Maintaining constant pressure in the liquid nitrogen supply is critical for steady, trouble-free operation. After this cooling run, a dedicated, pressure building tank was installed to maintain a constant pressure in all supply tanks.

\section{CONCLUSIONS}

The current APS cools large infrared instruments with an accurate and continuous rate of temperature change without relying on skilled operators. With improved supply tank management, the APS can very efficiently cool an instrument in the shortest possible time. This efficiency is achieved by reducing temperature rise during supply tank switching and tank replacement and operating continuously for 24 hours a day. Remote monitoring and control over a local network or the Internet give the operational team a high level of flexibility and comfort during a pre-cooling run. Work at the summit of Mauna Kea is reduced to one team member performing liquid nitrogen tank replacement once per day.

\section{REFERENCES}

[1] Ichikawa, T., Suzuki, R., Tokoku, C., Uchimoto, Y. K., Konishi, M., Yoshikawa, T., Yamada, T., Tanaka, I., Omata, K., Nishimura, T., "MOIRCS: multi-object infrared camera and spectrograph for SUBARU" in Ground-based and Airborne Instrumentation for Astronomy, edited by Ian S. McLean, Masanori Iye, Proceedings of SPIE Vol. 6269 (SPIE, Bellingham, WA 2006) 626916.

[2] Thomas M. Flynn, "Cryogenic Engineering”,Marcel Dekker, Inc.(1997), ISBN 0-8247-9724-8 